\title{
Synaptonemal complex formation in Avena polyploids
}
M. Jones,
$H$. Rees and
G. Jenkins

\author{
Department of Agricultural Botany, \\ University College of Wales, Aberystwyth, \\ Dyfed SY23 3DD, U.K.
}

\begin{abstract}
Avena maroccana $(2 n=4 x=28)$ and $A$. sativa $(2 n=6 x=42)$ are allopolyploids. Reconstructions from electron micrographs of synaptonemal complexes in serial sections of pollen mother cells showed that associations at zygotene and subsequent stages of meiosis are confined to homologous chromosomes only, with the result that only bivalents are generated. Such "classical" behaviour contrasts sharply with that in allopolyploids of wheat, Lolium and Scilla. In these pairing at zygotene involves homoeologous as well as homologous chromosomes, generating not only bivalents but multivalents which are "resolved" to bivalents prior to first metaphase.
\end{abstract}

An analysis is presented, also, of structural and genetic factors which influence the distribution of pairing within and between chromosomes at zygotene in the Avena species.

\section{INTRODUCTION}

The wild and cultivated species of Avena are represented by diploids, tetraploids and hexaploids with $2 n=14,28$ and 42 . The polyploids are of hybrid origin and chromosome behaviour at meiosis is typical of classical allopolyploids. Pairing at first metaphase is restricted to bivalents comprised of strictly homologous chromosomes. Chromosome segregation is regular and the fertility high. Inheritance is disomic (Thomas, 1989). The "classical" explanation for the diploid-like behaviour of allopolyploids is that homologous and homoeologous chromosomes are structurally so dissimilar that they fail to associate with one another at early prophase, i.e., at zygotene of meiosis. In at least some instances this explanation needs qualification.

1. In Triticum (Riley and Chapman, 1958; Sears and Okamoto, 1958) and in synthetic allopolyploids of Lolium (Evans and Macefield, 1973) structural differences between homologous and homoeologous chromosomes are not in themselves sufficient to ensure that only bivalents appear at first metaphase. "Diploidising" genes, located in the nucleus, are essential components of the system, serving as it were to reinforce the distinction between homologues and homoeologues.
2. While pairing at first metaphase in both Triticum and Lolium is strictly within pairs of homologous chromosomes, "pairing" at zygotene even with the presence of diploidising genes, is not. At this stage observations by electron microscopy in Triticum (Hobolth, 1981; Jenkins, 1983; Holm, 1986, 1988; Holm and Wang, 1988), in Lolium (Jenkins, 1985b, 1986) and in Scilla (Jenkins et al., 1988) show that multivalents are generated, involving both homologous and homoeologous chromosomes. These multiple configurations are subsequently "corrected" to bivalents. The correction mechanism may well be mediated by the diploidising genes.

The first question posed in this paper is whether the diploidising mechanism in Avena conforms to that in Triticum and Lolium, in particular whether there is multivalent formation at zygotene, homoeologous as well as homologous association. The second part of the paper deals with the finer details of chromosome associations at zygotene and with structural and other factors which influence their distribution.

\section{MATERIALS AND METHODS}

Seeds of the tetraploid $A$. maroccana $(2 n=4 x=$ $28)$ and the hexaploid $A$. sativa $(2 n=6 x=42)$ were 
obtained from the Welsh Plant Breeding Station. Plants were grown in a heated greenhouse.

\section{Fixation}

Anthers with pollen mother cells at the zygotene/pachytene stage of meiosis were immersed in 4 per cent glutaraldehyde in $0 \cdot 1 \mathrm{M}$ phosphate buffer and dehydrated in a graded ethanol series. The anthers were stained in 1 per cent phosphotungstic acid (PTA) in ethanol for 16 hours at $4^{\circ} \mathrm{C}$ and then washed in ethanol. The anthers were infiltrated with Spurr low-viscosity resin on a rotator and embedded in moulds and polymerised in an oven at $70^{\circ} \mathrm{C}$.

\section{Sectioning}

Transverse thick survey sections were cut from each block using a Reichert OMU4 "Ultracut" microtome to check the meiotic stage of the anthers and degree of fixation damage. Two sections 1$2 \mu \mathrm{m}$ thick were cut using a microtome equipped with a glass knife. The sections were examined under phase contrast and the most suitable loculus in each anther was identified. Several ultra-thin $(100 \mathrm{~nm})$ sections were cut from each locule with a glass knife. Sections were floated onto distilled water, flattened with a heat pen and picked up in a drop of water in the centre of a $2 \times 1 \mathrm{~mm}$ copper slot grid. They were transferred to carbon coated Formvar support film stretched across a plastic ring and allowed to dry (Wells, 1974). These sections were examined under a Jeol JEM $100 \mathrm{CX}$ electron microscope at $80 \mathrm{kV}$. If they were suitable a series of sections, $100 \mathrm{~nm}$ thick, was cut through the selected locule using a $3 \mathrm{~mm}$ "Diatome" diamond knife. Each section within a nucleus was photographed sequentially at a nominal magnification of 3300 .

\section{The process of $3 D$ reconstruction}

Unpaired axial cores and synaptonemal complexes (SCs) were traced through the micrographs spanning the nucleus, and numbered. The positions of the following features were recorded: the centromeres, the nucleolar organizing regions, blocks of heterochromatin, the telomeres and whether the latter were attached or unattached. These were traced onto acetate sheets, providing information about the disposition of the chromosomes in three dimensions. The lengths of the chromosomes were measured using a Bausch and Lomb "Hipad" digitiser, recorded and plotted on a $\mathrm{BBC}$ micro- computer. Full details of the fixation, sectioning and reconstruction procedures are given by Jenkins (1985a).

Serial sectioning of nuclei was chosen in preference to spreading in the belief that the chromosomes were less liable to fracture during handling. The belief was unjustified.

\section{RESULTS}

\section{Distribution of pairing between chromosomes}

\section{Avena maroccana}

\section{Pachytene}

Fig. 1 represents the synaptonemal complexes in a pachytene nucleus. They comprise 14 separate configurations. In terms of relative length and arm ratio they correspond closely with the karyotype established from mitotic metaphases (Rajhathy, 1971). It is clear that pairing at this stage is between homologous chromosomes and restricted to homologous chromosomes.

One of the bivalents (4) has a large unpaired, interstitial segment. Bivalent 1 was broken. The acentric fragment could easily be identified because all the other chromosomes were intact. Fig. 1 also gives information concerning the attachment of ends to the nuclear membrane, the distribution of heterochromatin etc. It will be observed from fig. 1 that nucleoli are attached to bivalents 1 and 2 only. In somatic regions, e.g. in root meristems, nucleolar organisers (NOR's) are active in three pairs of chromosomes. Suppression of NOR activity in pollen mother cells has also been reported in Lolium (Jenkins, 1985a).

\section{Zygotene}

Reconstructions of two zygotene nuclei $(Z 1$ and $Z 2$ ) are represented in figs. 2(a) and 2(b). About 48 per cent of the lateral elements are paired in $Z 1$, about 39 per cent in $Z 2$.

In both nuclei the associations are between homologous chromosomes and strictly confined to homologous chromosomes. Details of attachments to nuclear membranes, heterochromatic segments etc. are given in the figures. It will be observed that there is some variation between $Z 1$ and $Z 2$ in the degree of attachment of telomeres to the nuclear membrane and in the manifestation of heterochromatic segments in particular chromosomes. Both, like the pachytene nucleus, show NOR activity in only two of the bivalents. 


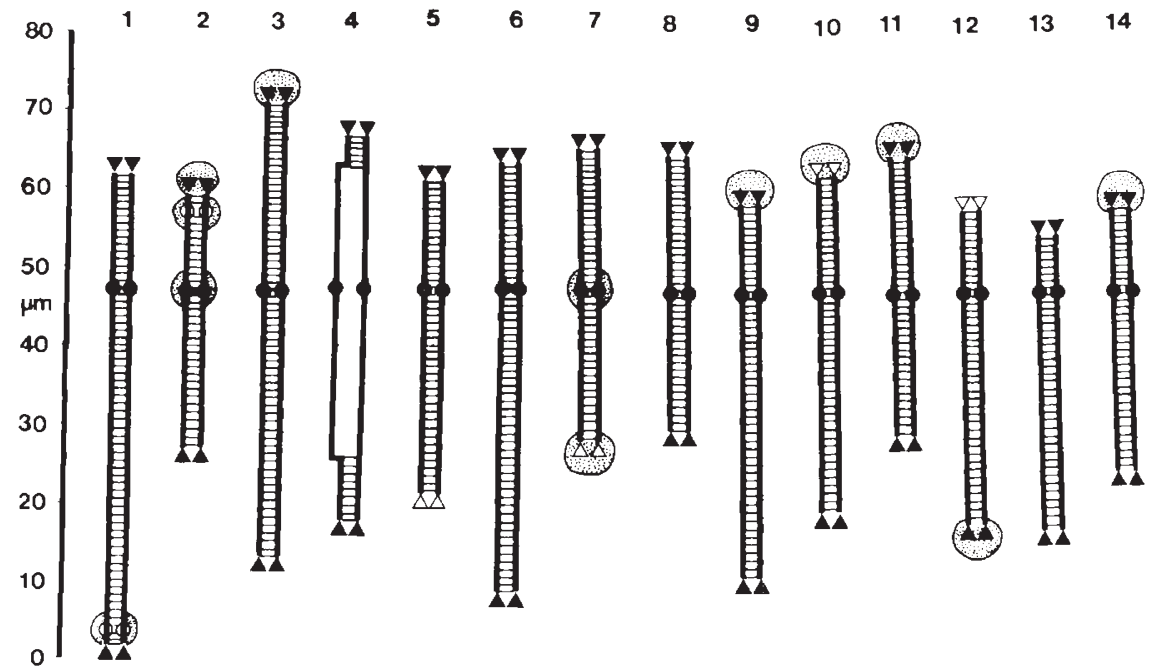

Figure 1 Reconstruction of the synaptonemal complexes at pachytene in Avena maroccana. Centromeres, solid circles; ends attached to the nuclear membrane, solid triangles; ends not attached, open triangles; heterochromatin, dotted circles; open circles, nucleolar organisers. Cross hatching denotes pairing. Arrows point to broken ends.

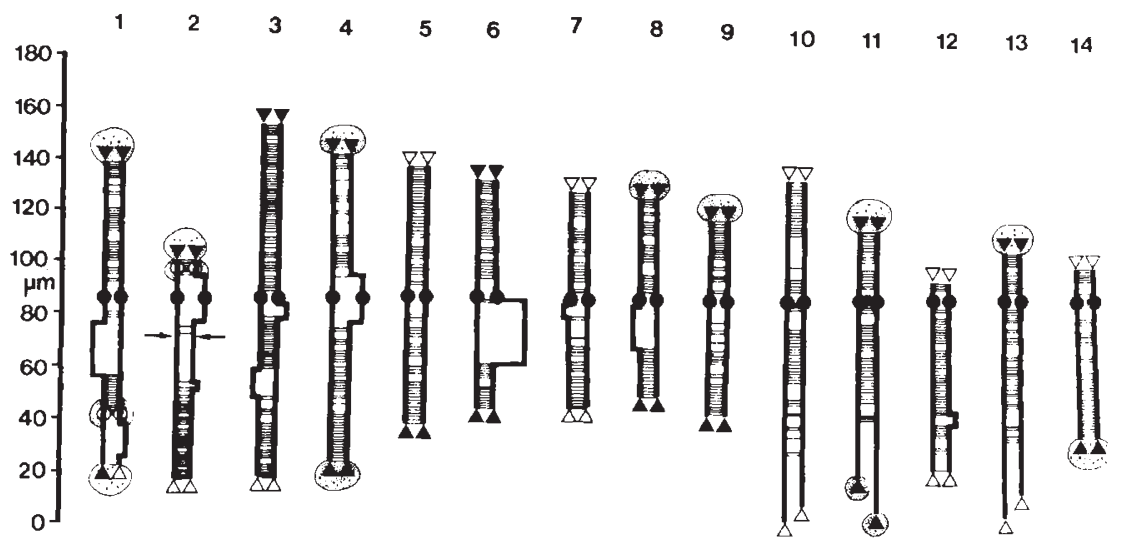

(a)

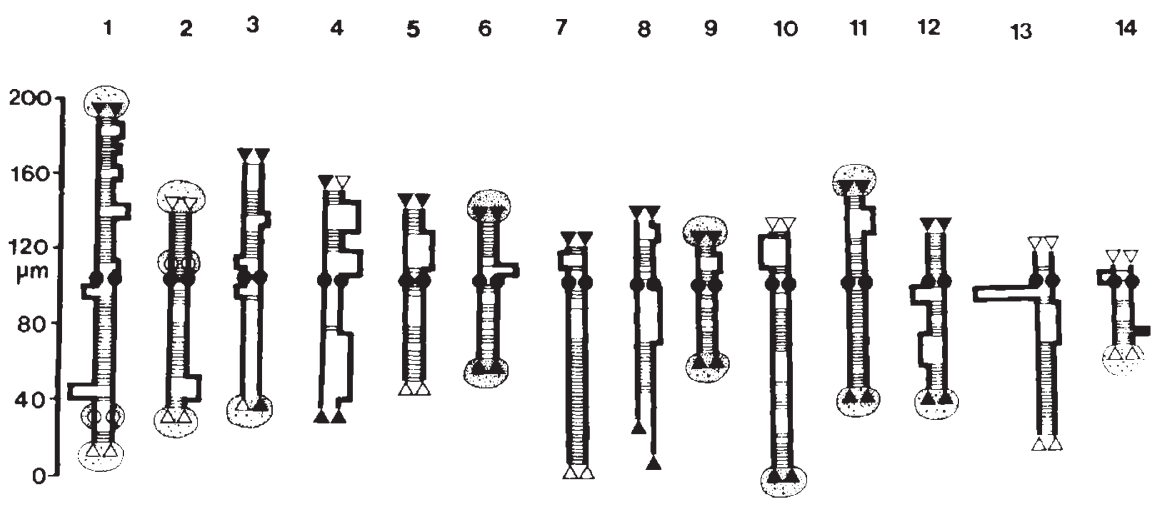

(b)

Figure 2 Reconstructions of synaptonemal complexes at zygotene in two nuclei, $Z 1$ (a) and $Z 2$ (b) in Avena maroccana. Labelling as in fig. 1. 


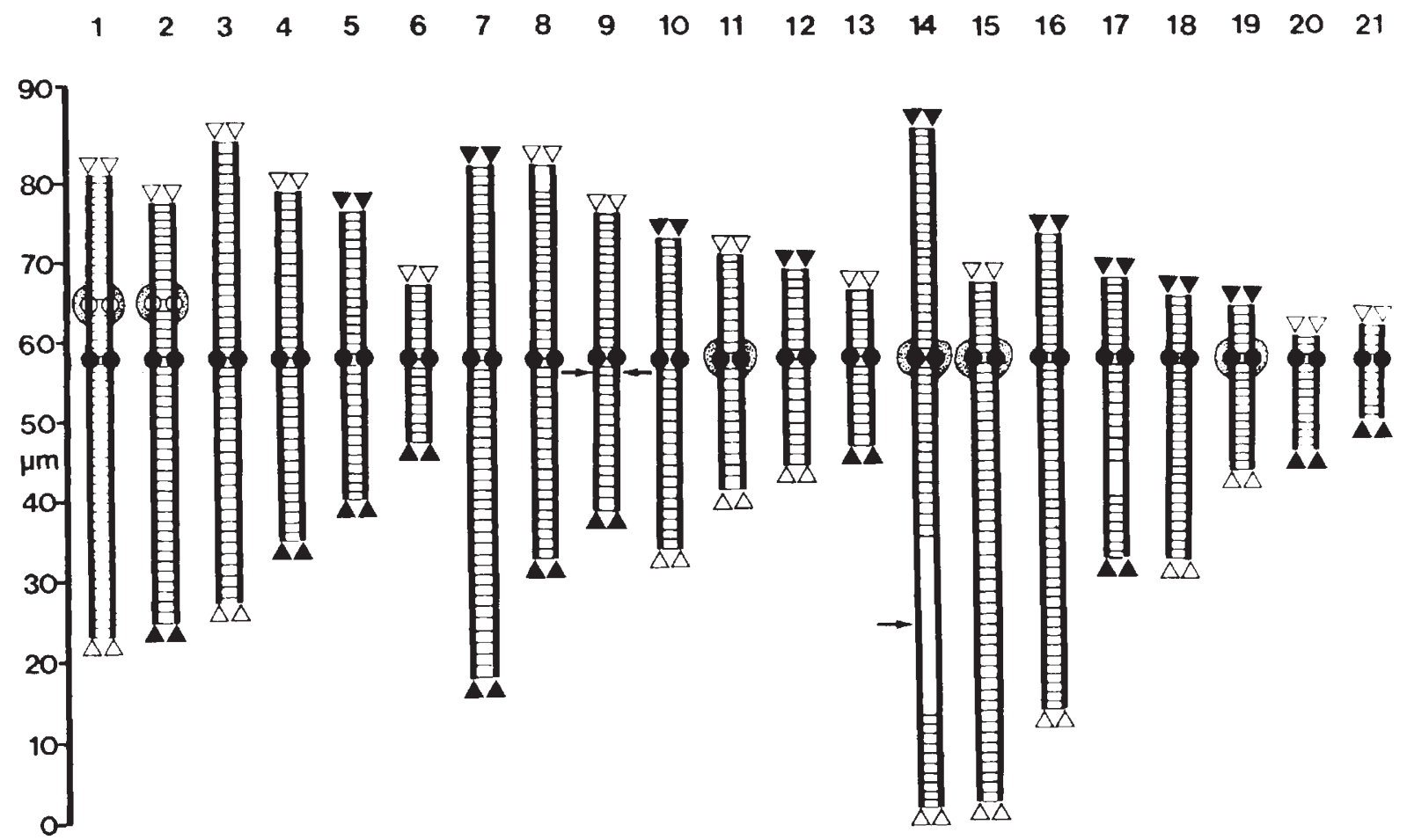

Figure 3 Reconstruction of the synaptonemal complexes at pachytene in Avena sativa. Labelling as in fig. 1.

\section{Avena sativa}

\section{Pachytene}

The reconstruction in fig. 3 shows 21 separate configurations, representing the 21 bivalents of the complement. Shapes and relative sizes correspond to those in the mitotic karyotype given by Rajhathy (1963). Bivalent 14 has an unpaired segment in the long arm. Bivalent 9 is broken. The acentric fragment lay close to the break and was readily assignable to that bivalent. The NORs in 1 and 2 are active. One NOR, normally active in somatic cells, is inactive. Details of telomeric attachments etc. are given in fig. 3.

\section{Zygotene}

Synapsis is about 34 per cent complete (fig. 4). Unfortunately there was a substantial amount of fragmentation and only ten bivalents were entire. In eight of the eleven broken bivalents the acentric fragments could, with some confidence, be matched with their centric counterparts. There is no sign of multivalent formation. Only bivalents are generated. As in the pachytene nucleus there is evidence of NOR inactivation, but of a different kind. NORs produce nucleoli in each of three bivalents (1,2 and 3$)$ but the nucleoli are produced by NOR's in only one of each pair of homologous chromosomes (cf. Jenkins, 1985a). To summarise, in both the hexaploid $A$. sativa and the tetraploid A. maroccana the reconstructions of synaptonemal complexes, both at pachytene and zygotene, showed bivalent associations only, no trace of multivalents.

\section{Distribution of pairing within chromosomes}

Below is a description and analysis of the distribution of pairing within bivalents of the two zygotene nuclei of $A$. maroccana. The procedure was as follows. Each bivalent was divided into six equal sections, 1 to 6 (tables 1 and 2). In this connection there was a problem in estimating the total length of each bivalent. In figs. 2(a) and (b) it will be seen that the lateral elements in unpaired regions are frequently of different length due no doubt to different degrees of contraction. In such regions the shorter of the two elements was chosen in estimating the total length of each bivalent. This seems reasonable on the grounds that the lateral elements are in process of contraction as they associate to form the synaptonemal complexes. The total was then divided into six sections. In 
$\begin{array}{llllllllllllll}1 & 2 & 3 & 4 & 5 & 6 & 7 & 8 & 9 & 10 & 11 & 12 & 13 & 14\end{array}$

$\begin{array}{lll}16 & 17 & 18\end{array}$

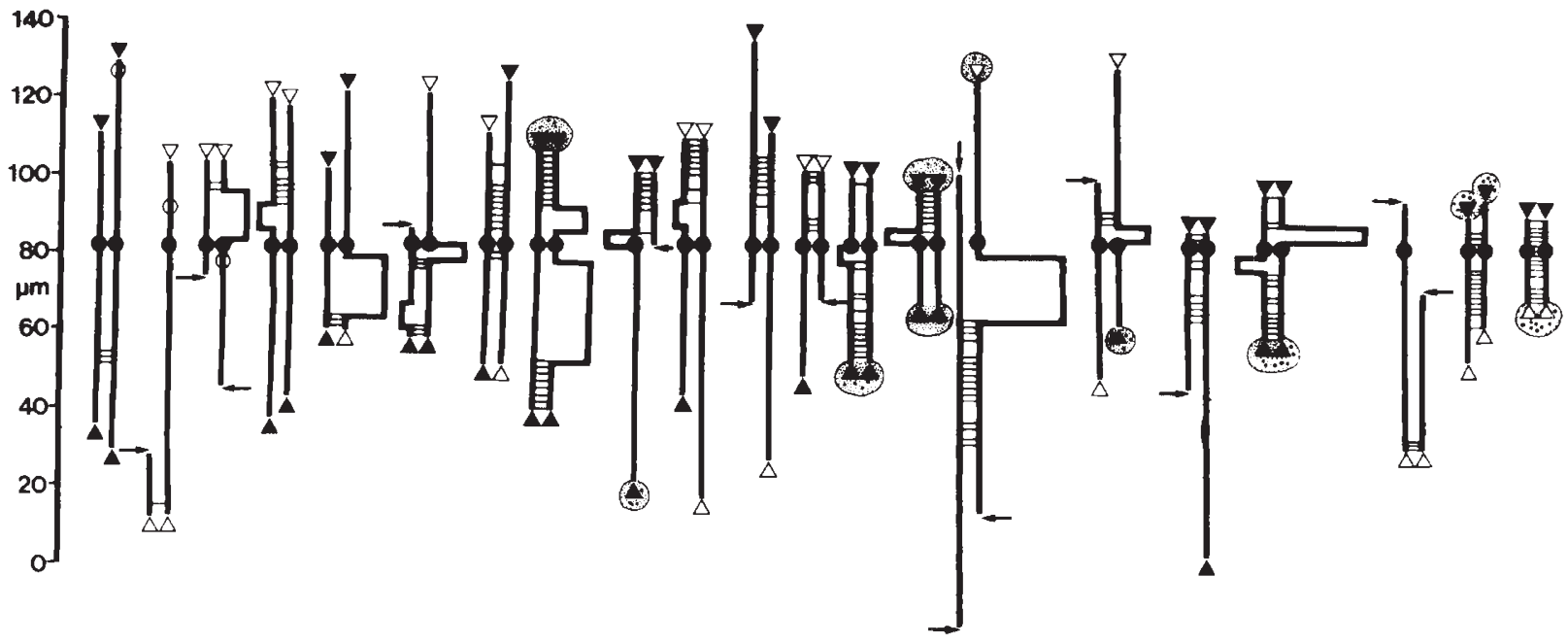

Figure 4 Reconstruction of the synaptonemal complexes and lateral elements in a zygotene nucleus in Avena sativa. Arrows point to broken ends. Unpaired fragments omitted.

Table 1 The distribution of pairing at zygotene in $Z 1$. Lengths paired in each section in microns. Values in brackets are proportions paired in angular values. Short arms to the left

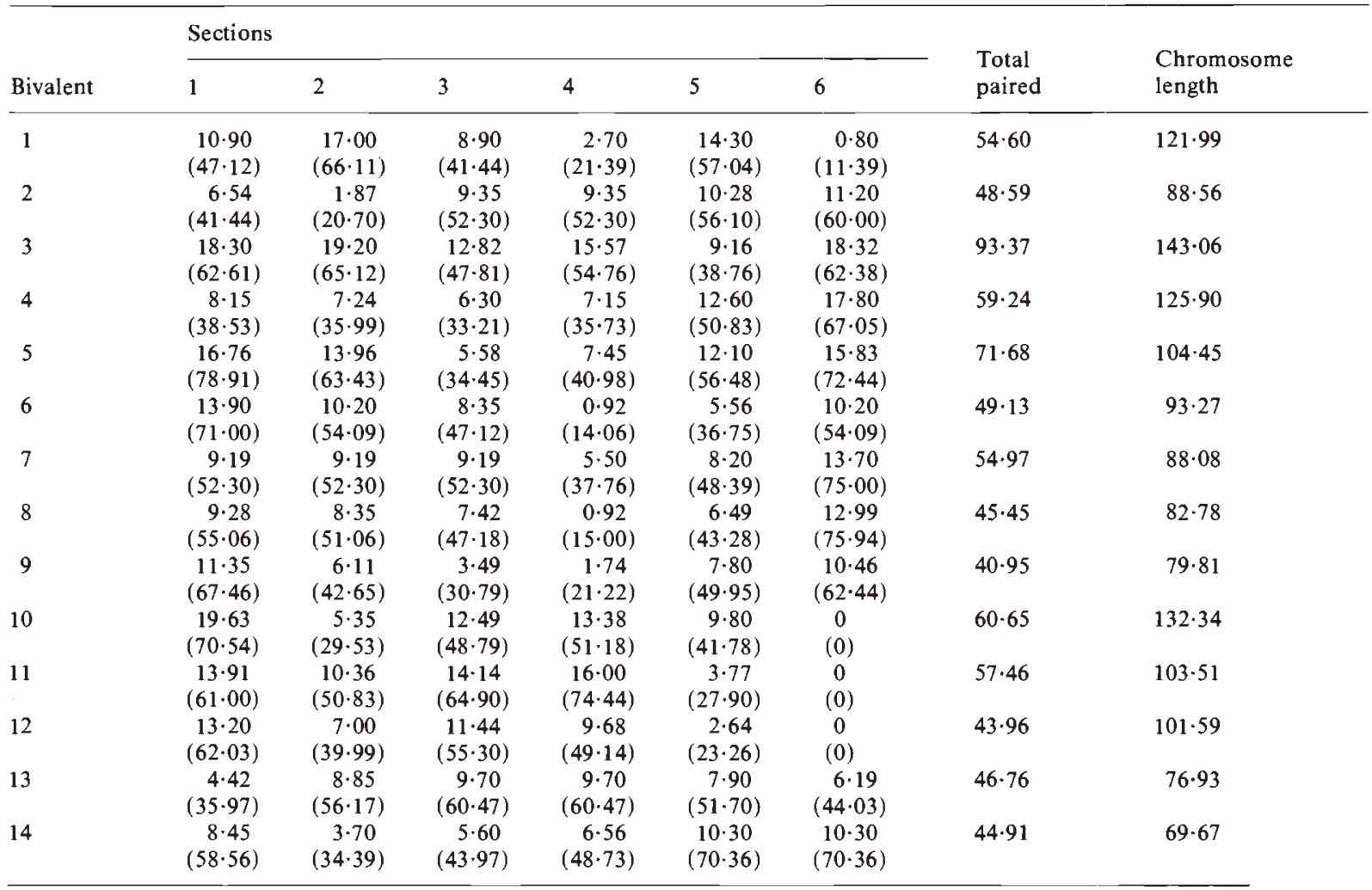


Table 2 The distribution of pairing at zygotene in Z,2. Lengths paired in each section in microns. Values in brackets are proportions paired in angular values. Short arms to the left

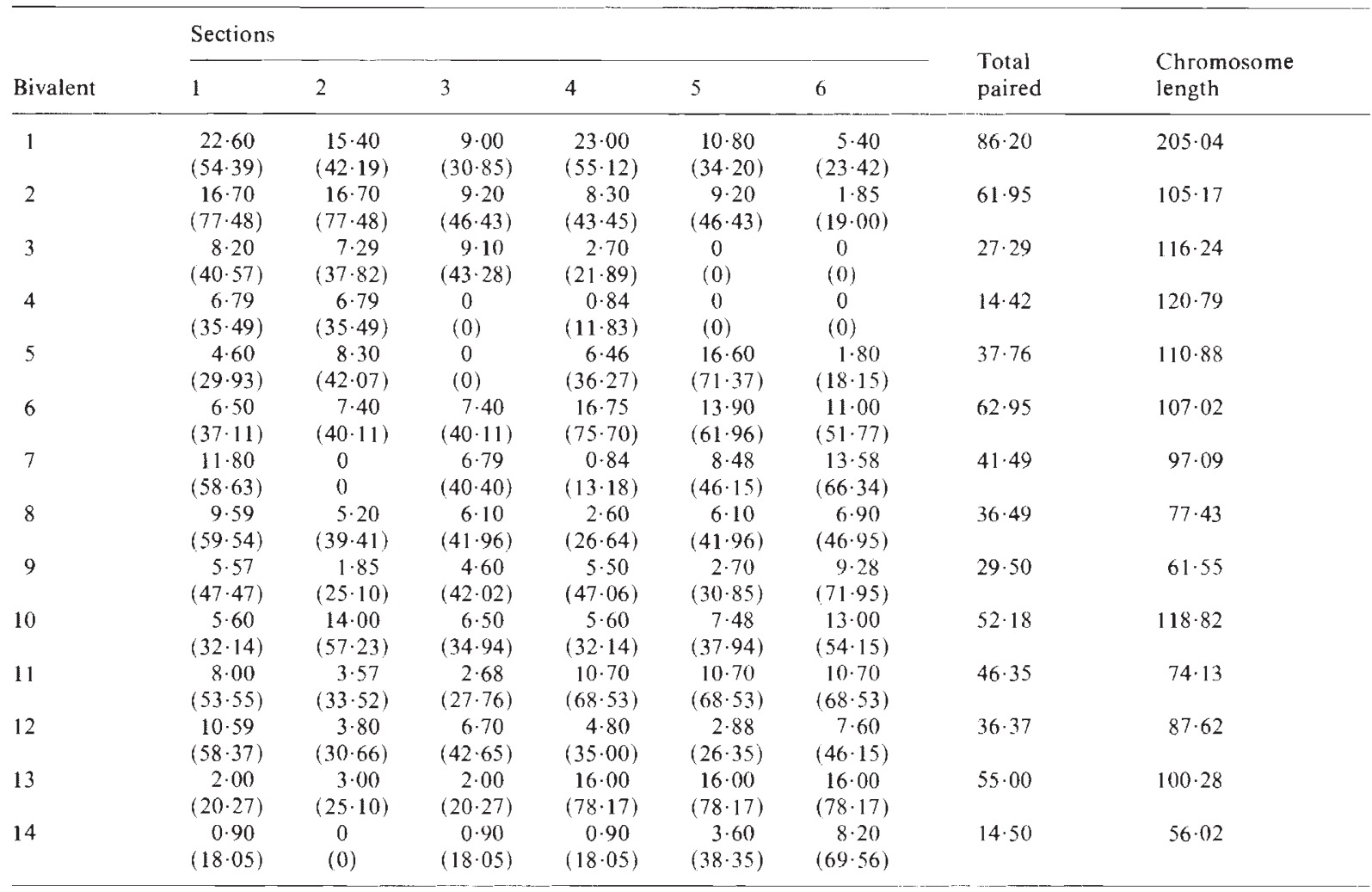

tables 1 and 2 the length paired are recorded in each section and the proportion paired in each. The latter are expressed as angular values for purposes of analysis.

It is important to emphasise that the order in which the chromosomes are listed and numbered in $Z 1$ (i.e., in table 1 and fig. 2(a)) do not necessarily correspond with those in $Z 2$ (i.e., in table 2 and fig. 2(b)). The variation in total length of synaptonemal complexes and lateral elements and in arm ratios is influenced so much by the amount and distribution of pairing in particular that only a small number of bivalents could be identified and matched with confidence in the two nuclei.

\section{Variation between sections}

The analysis of variance in table 3 (a) shows that, within nuclei, the amount of pairing in the end sections ( 1 and 6 ) is significantly greater than in the middle sections (2, 3, 4 and 5). The conclusion is that pairing is initiated preferentially at the ends of the chromosomes rather than in the middle regions (fig. 5). From the configurations in figs. 2(a) and 2(b) the absence of pairing in the end segments of a number of bivalents (e.g., 1, 10, 11 and 13 in 2(a); 3, 4, 8 and 13 in 2(b)) would appear to conflict with the conclusion of pairing initiation in the end sections. The conflict is only apparent. Pairing is initiated at one end or the other is some bivalents (e.g. 3 and 13 in 2(b)); at both ends in others (fig. 6). Table 3 shows, also, that there is no significant interaction between the pairing pattern (ends versus middle sections) and nuclei.

\section{The centromere section}

Given that pairing is initiated at the ends of the chromosomes it is to be expected that pairing in the sections containing the centromeres should be relatively low because the centromeres are located in median or sub-median sections. One may ask, however, whether the centromere in itself influences the amount of pairing in the section in which it is contained. The question may be answered by comparing the amount of pairing in the centromere section with the average of the amount of pairing 
Table 3

(a) The analysis of variance of differences in the amount of pairing between the end and middle sections of chromosomes in $Z 1$ and $Z 2$

\begin{tabular}{|c|c|c|c|c|c|}
\hline Item & SS & $N$ & MS & VR & $P$ \\
\hline Between $1+6$ and $3+4+5+6$ & $1795 \cdot 19$ & 1 & $1795 \cdot 19$ & $5 \cdot 82$ & 0.05 \\
\hline Between $1+6$ and $3+4+5+6 \times$ nuclei & $7 \cdot 89$ & 1 & $7 \cdot 89$ & & \\
\hline Error & $8021 \cdot 43$ & 26 & $308 \cdot 52$ & & \\
\hline
\end{tabular}

(b) The analysis of variance of differences in the proportion of total lengths paired between chromosomes

\begin{tabular}{|c|c|c|c|c|c|}
\hline Item & SS & $N$ & MS & VR & $P$ \\
\hline Between nuclei & $3018 \cdot 80$ & 1 & $3018 \cdot 80$ & & \\
\hline Between chromosomes within nuclei & $13143 \cdot 51$ & 26 & $505 \cdot 52$ & $1 \cdot 41$ & $\mathrm{n} \leqslant$ \\
\hline Error & $50106 \cdot 26$ & 140 & $357 \cdot 90$ & & \\
\hline
\end{tabular}

in the two sections on either side. By choosing sections on each side of the centromere one has a reliable estimate of the amount of pairing in that region of the chromosome be it high or low, late or early in the pairing process. Table 4 shows the amount of pairing in the centromere section and the mean pairing in adjacent sections for 13 bivalents in $Z 1$ and 12 bivalents in $Z 2$. Bivalents in which the centromere embraces two sections have been omitted. An analysis of the differences between the amount of pairing in the centromere and adjacent sections for all bivalents in each of the two nuclei (see Mather, 1943) shows no significance $(t=0 \cdot 91 ; N=24 ; P=0 \cdot 3-0 \cdot 5)$. We con-

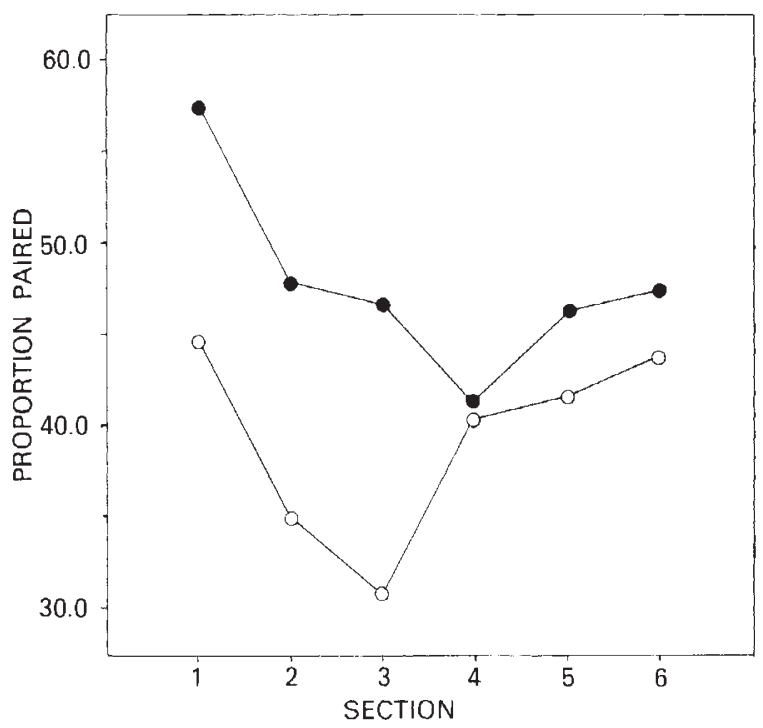

Figure 5 The average proportion -aired (angular values) in sections 1 to 6 of chromosomes in $Z 1$ (solid circles) and $Z 2$ (open circles). clude that the centromeres have no effect on pairing in their immediate vicinity.

\section{Variation between chromosomes}

The analysis of variance in table $3(\mathrm{~b})$ shows no difference in the proportion of total length paired between chromosomes. Because the chromosomes within complements are of different lengths this implies however that, in absolute terms, the total

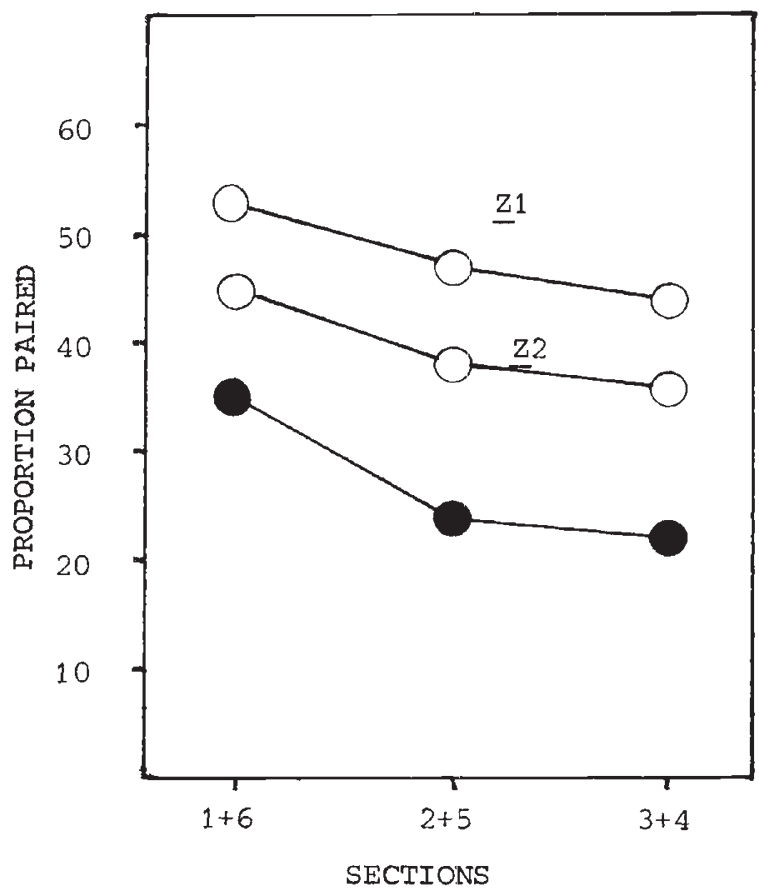

Figure 6 The proportion paired at zygotene in end sections $(1+6)$, sections $2+5$ and $3+4$ in $Z 1, Z 2$ and in the $A$. sativa nucleus (solid circles). 
Table 4 The amount of pairing in the sections containing the centromere and the mean amount of pairing in adjacent sections (in angles) in bivalents from $Z_{1}$ (a) and $Z_{2}$ (b)

\begin{tabular}{|c|c|c|c|c|}
\hline \multirow[b]{2}{*}{ Bivalent } & \multicolumn{2}{|l|}{ (a) } & \multicolumn{2}{|l|}{ (b) } \\
\hline & $\begin{array}{l}\text { Centromere } \\
\text { section }\end{array}$ & $\begin{array}{l}\text { Mean of adjacent } \\
\text { sections }\end{array}$ & $\begin{array}{l}\text { Centromere } \\
\text { section }\end{array}$ & $\begin{array}{l}\text { Mean of adjacent } \\
\text { sections }\end{array}$ \\
\hline 1 & $41 \cdot 44$ & $43 \cdot 75$ & $30 \cdot 85$ & $48 \cdot 55$ \\
\hline 2 & $20 \cdot 70$ & $48 \cdot 87$ & 46.43 & $60 \cdot 47$ \\
\hline 3 & $47 \cdot 81$ & $59 \cdot 94$ & $43 \cdot 28$ & $29 \cdot 86$ \\
\hline 4 & $33 \cdot 21$ & $35 \cdot 85$ & 0 & $23 \cdot 66$ \\
\hline 5 & 34.45 & $52 \cdot 21$ & $42 \cdot 07$ & 14.97 \\
\hline 6 & $47 \cdot 12$ & $34 \cdot 08$ & $40 \cdot 11$ & 57.91 \\
\hline 7 & $52 \cdot 30$ & $45 \cdot 03$ & $40 \cdot 40$ & 6.59 \\
\hline 8 & $47 \cdot 18$ & $33 \cdot 03$ & $41 \cdot 96$ & $33 \cdot 03$ \\
\hline 9 & $30 \cdot 79$ & $36 \cdot 94$ & $42 \cdot 02$ & $36 \cdot 08$ \\
\hline 10 & $48 \cdot 79$ & $40 \cdot 36$ & - & - \\
\hline 11 & $50 \cdot 83$ & $62 \cdot 95$ & $33 \cdot 52$ & $40 \cdot 65$ \\
\hline 12 & - & - & $30 \cdot 66$ & $50 \cdot 51$ \\
\hline 13 & - & - & - & - \\
\hline 14 & $34 \cdot 39$ & $51 \cdot 27$ & $18 \cdot 05$ & $9 \cdot 03$ \\
\hline Means & $40 \cdot 75$ & $45 \cdot 19$ & $34 \cdot 11$ & $34 \cdot 29$ \\
\hline
\end{tabular}

amount of pairing in each chromosome varies directly with length. This is confirmed by the graphs in fig. 7. The longer the chromosome the more the pairing. The joint regression is significant $(P=<0.01)$ and there is no heterogeneity between the slopes for $Z 1$ and $Z 2$. Three explanations are possible. The first is that the rate at which the pairing "spreads" from the points of initial contact is faster in the long chromosomes as compared with the shorter ones. The second is that the long chromosomes start pairing before the shorter chromosomes. The third is that there are more pairing initiation sites in the longer chromosomes. From our results there are no means for deciding between the alternatives. Even so it is worth mentioning that whatever the cause the consequence is important, namely that completion of pairing may be achieved in synchrony and irrespective of variation in chromosome length within the complement.

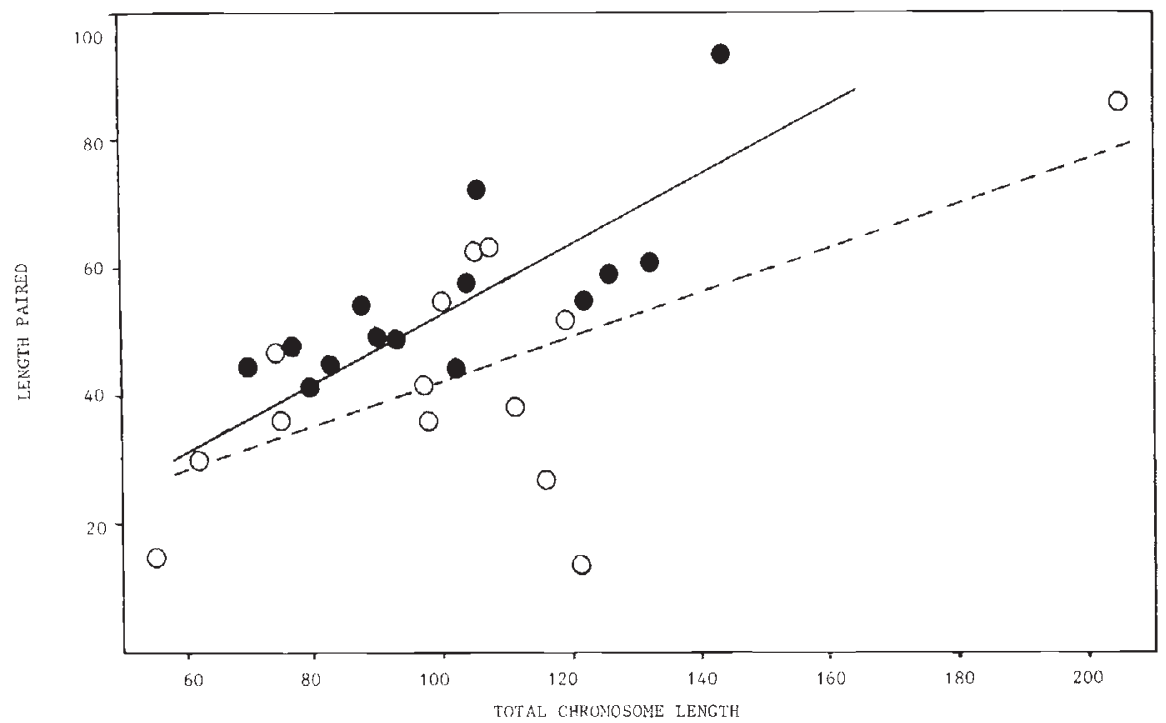

Figure 7 The total amount of pairing plotted against chromosome lengths (in microns) in $Z 1$ and $Z 2$. Solid circles and line $Z 1$; open circles and dotted line $Z 2$. 
Table 3 confirms also a significant difference between the mean square between nuclei when tested against the mean square between chromosomes within nuclei.

\section{Heterochromatin}

Figs. 2(a) and 2(b) show terminal blocks of heterochromatin in many of the bivalents. It is pertinent to inquire whether they affect pairing at this zygotene stage. The amount of pairing in terminal sections with and without heterochromatin are given in table 5. An analysis of variance shows no significant variation due to heterochromatin. While heterochromatic segments are devoid of chiasmata at later stages of meiosis these results confirm that their absence is not attributable to failure of pairing at zygotene.

\section{Heterochromatin and telomere attachment}

In both $Z 1$ and $Z 2$ either one or both telomeres in about 60 per cent of the chromosome arms is attached to the nuclear membrane. In both nuclei telomeres with heterochromatin are attached to the nuclear membrane at a higher frequency than telomeres without heterochromatin (figs. 2(a) and 2 (b) and table 5). The difference is significant $\left(\chi^{2}(1)=11.73 ; P=<0 \cdot 001\right)$. It could be that heterochromatin facilitates attachment. Alternatively attachment may cause the heterochromatin to be more clearly expressed. Certainly its expression varies between nuclei. The amount of pairing in the terminal sections is not affected by attachment to the nuclear membrane. This is to be expected from the fact that attachment is clearly correlated with the presence of heterochromatin which, in turn, has no influence on pairing.

In view of the incompleteness of the zygotene nucleus reconstructed from $A$. sativa no detailed analysis of the distribution of pairing was attempted. It is clear from fig. 6, however, that pairing, as in $A$. maroccana, was initiated mainly at the ends of the chromosomes.

\section{DISCUSSION}

\section{Diploidisation}

In hexaploid wheat and in the synthetic L.temulentum $\times$ L. perenne allopolyploids, diploidisation, as described earlier, is under the control of nuclear genes. It will be recalled that these diploidising genes do not prevent homoeologous pairing or multivalent formation at zygotene, although, there is an indication that they do suppress the frequency of multivalents (Holm and Wang, 1988). In contrast our results suggest that diploidisation in Avena is achieved by complete suppression of homoeologous pairing. None of the three zygotene nuclei contain multivalents. In wheat two nuclei at mid zygotene examined by Hobolth (1981) both

Table 5 The amount of pairing (in angular values) in terminal sections (1 and 6) with and without heterochromatin

\begin{tabular}{|c|c|c|c|}
\hline \multicolumn{2}{|l|}{$Z 1$} & \multicolumn{2}{|l|}{$Z 2$} \\
\hline With heterochromatin & Without & With heterochromatin & Without \\
\hline $\begin{array}{r}47 \cdot 12^{\mathrm{A}} \\
41 \cdot 44^{\mathrm{A}} \\
38 \cdot 53^{\mathrm{A}} \\
55 \cdot 06^{\mathrm{A}} \\
67 \cdot 46^{\mathrm{A}} \\
61 \cdot 00^{\mathrm{A}} \\
62 \cdot 03^{\mathrm{A}} \\
11 \cdot 39^{\mathrm{A}} \\
67 \cdot 05^{\mathrm{A}} \\
70 \cdot 36^{\mathrm{A}} \\
\frac{0^{\mathrm{A}}}{47 \cdot 40}\end{array}$ & $\begin{array}{c}62 \cdot 31^{\mathrm{A}} \\
78 \cdot 91 \\
71 \cdot 00^{\mathrm{A}} \\
52 \cdot 30 \\
70 \cdot 54 \\
35 \cdot 97 \\
58 \cdot 56 \\
60 \cdot 00 \\
62 \cdot 38 \\
75 \cdot 00 \\
54 \cdot 09^{\mathrm{A}} \\
72 \cdot 44^{\mathrm{A}} \\
62 \cdot 44^{\mathrm{A}} \\
0 \\
0 \\
44.03 \\
75 \cdot 94^{\mathrm{A}} \\
55 \cdot 05\end{array}$ & $\begin{array}{c}54 \cdot 39^{\mathrm{A}} \\
77 \cdot 48 \\
37 \cdot 11^{\mathrm{A}} \\
59 \cdot 54^{\mathrm{A}} \\
47 \cdot 47^{\mathrm{A}} \\
23 \cdot 42 \\
19 \cdot 00 \\
0^{\mathrm{A}} \\
51.77^{\mathrm{A}} \\
46.95^{\mathrm{A}} \\
71.95^{\mathrm{A}} \\
54.15^{\mathrm{A}} \\
46 \cdot 15^{\mathrm{A}} \\
69 \cdot 56 \\
67.07\end{array}$ & $\begin{array}{c}40 \cdot 57^{\mathrm{A}} \\
35 \cdot 49^{\mathrm{A}} \\
29 \cdot 93^{\mathrm{A}} \\
58 \cdot 63^{\mathrm{A}} \\
32 \cdot 14 \\
53 \cdot 55 \\
58 \cdot 37 \\
20 \cdot 27 \\
18 \cdot 05 \\
0^{\mathrm{A}} \\
18 \cdot 15^{\mathrm{A}} \\
66 \cdot 34 \\
68 \cdot 53 \\
\underline{78 \cdot 17} \\
\text { Mean } 41 \cdot 29\end{array}$ \\
\hline
\end{tabular}

\footnotetext{
A , denotes telomeres attached to the nuclear membrane.
} 
contained multivalents. An early zygotene nucleus reconstructed by Jenkins (1983) also showed multivalent formation. Eight out of eleven nuclei at mid-zygotene analysed by Holm (1986) contained multivalents (late zygotene nuclei may not be strictly comparable because of the possibility that some multivalents are eliminated at this stage). In Lolium, similarly, multivalents were reported in each of the two nuclei reconstructed at early zygotene in an allotetraploid (Jenkins, 1986) and at early zygotene in an allotriploid (Jenkins, 1985b). The Avena sample is, unfortunately, pitifully small. Even so the three reconstructions at zygotene suggest that homoeologous pairing and multivalent formation is, at the very least, much reduced in comparison with wheat and Lolium. It is worth considering why this should be so.

\section{Chromosome homology}

In hybrids between the putative diploid ancestors of Triticum aestivum there is a relatively high degree of pairing and chiasma formation at first metaphase of meiosis (Sears, 1941; Kimber and Riley, 1963). In the diploid hybrid between $L$. temulentum and $L$. perenne, also, pairing and chiasma formation, in the absence of diploidising genes, are of a high order. Evidently there is a considerable degree of structural similarity between the homoeologous chromosomes of the diploid ancestors in both wheat and Lolium. The situation in Avena is different. While the ancestry of Avena polyploids is less well established it is generally agreed that $A$. maroccana derives from diploids of the genomic constitution $A A$ and $C C$ respectively (Rajhathy and Thomas, 1974). Hybrids between $A A$ and $C C$ diploids are difficult to make, suggesting considerable genetic diversity between them. Only one $A C$ hybrid has been described (Nishiyama and Yabuna, 1975), that between $A$. strigosa $(A A)$ and $A$. pilosa $(C C)$. Pairing at first metaphase in the hybrid is very low with an average of less than one bivalent per pollen mother cell. On the face of it the $A$ and $C$ chromosomes are structurally very dissimilar (see also Rajhathy and Dyck, 1963; Rajhathy and Thomas, 1967, 1974). A. sativa, like A. maroccana, contains the genomes $A A$ and $C C$. The origin of the $D D$ genome is unknown.

From these observations the distinction between wheat and Lolium, on the one hand, and Avena on the other, could be explained as follows. In wheat and Lolium allopolyploids the structural similarity between ancestral genomes is such that homoeologous pairing and multivalent formation at zygotene is inevitable, even in the presence of diploidising elements. In the Avena polyploids the structural divergence between ancestral genomes is such that there is little homology between them, little or no homoeologous pairing or multivalent formation at zygotene. One might even argue that the structural diversity is so great that there is no "requirement" for diploidising genes to ensure bivalent formation at first metaphase. This argument is, however, questionable.

\section{Diploidising elements in Avena}

The evidence for the very substantial structural diversity between the $\mathrm{A}$ and $\mathrm{C}$ genomes is that chromosome pairing in the AC hybrid is low. Suppose, however, that the AA or CC diploid parents, or both, carried diploidising genes. The evidence then would be invalid. There is certainly evidence that Avena polyploids carry diploidising genes; so may the diploids. In a polyhaploid $A$. sativa the loss of a particular chromosome results in considerable amount of pairing between A, C and D chromosomes (Gauthier and McGinnis, 1968; Leggett, 1977). The missing chromosome presumably carries a diploidising gene or genes. In the F1 hybrid between $A$. sativa and $A$. longiglumis (strain CW57) there is also pairing between $\mathrm{A}, \mathrm{C}$ and $\mathrm{D}$ chromosomes, suggesting that $A$. longiglumis carries suppressors of diploidising elements located in A. sativa (Rajhathy and Thomas, 1972).

The suppression of homoeologous pairing in Avena allopolyploids is not, therefore, explicable on grounds of structural diversity between the ancestral genomes alone. Either the diploidising elements in Avena are more effective per se in preventing pairing between homoeologous chromosomes at zygotene or, else, they are more effective because the diversity between ancestral genomes in Avena, in relative terms, is that much greater than is the case for wheat and Lolium. It is of interest to draw attention to the recent work on diploidisation in the allotetraploid Allium montanum (Loidl, 1988). As in Avena pairing at zygotene is restricted to homologous chromosomes only.

\section{The distribution of pairing within bivalents}

Events at zygotene clearly are of significance to the subsequent formation and distribution of chiasmata. In the Avena species our observations show that pairing is initiated towards the ends of the chromosomes. These are the very regions in which chiasmata are concentrated. It is tempting 
to invoke a causal relation. An element of caution, however, is called for. Pairing in heterochromatic regions, it will be recalled, is effective at zygotene, yet chiasmata do not form in heterochromatic regions. While, therefore, the information about the distribution of pairing at zygotene is of interest and importance the precise significance of the pairing pattern with respect to chiasma formation remains conjectural.

Acknowledgements We acknowledge with gratitude the support of AFRC for this work. Also, we thank Dr A. Durrant for advice on statistical matters.

\section{REFERENCES}

EVANS, G. M. AND MACEFIELD, A. J. (1973). The effect of B chromosomes on homoeologous pairing in species hybrids. 1. Lolium temulentum $\times$ Lolium perenne. Chromosoma, 41 , 63-73.

GAUTHIER, F. M. AND McGinnis, R. C. (1968). The meiotic behaviour of a nulli-haploid plant in Avena sativa L. Can. J. Genet. Cytol, 10, 186-189.

HOBOLTH, P. (1981). Chromosome pairing in allohexaploid wheat var. Chinese Spring. Transformation of multivalents into bivalents, a mechanism for exclusively bivalent formation. Carlsberg Res. Commun., 46, 129-173.

HOLM, P. B. (1986). Chromosome pairing and chiasma formation in allohexaploid wheat, Triticum aestivum analysed by spreading of meiotic nuclei. Carlsberg Res. Commun., 51, 239-294.

HOLM, P. B. (1988). Chromosome pairing and synaptonemal complex formation in hexaploid wheat, monoisosomic and diisosomic for the long arm of chromosome 5B. Carlsberg Res. Commun., 53, 111-133.

HOLM, P. B. AND WANG, X. (1988). The effect of chromosome $5 \mathrm{~B}$ on synapsis and chiasma formation in wheat, Triticum aestivum cv. Chinese Spring. Carlsberg Res. Commun., 53, 191-208.

JENKINS, G. (1983). Chromosome pairing in Triticum aestivum cv. Chinese Spring. Carlsberg Res. Commun., 48, 255-283.

IENKINS, G. $(1985 a)$. Synaptonemal complex formation in hybrids of Lolium temulentum $\times$ Lolium perenne. I. Diploid. Chromosoma, 92, 81-88.

JENKINS, G. (1985b). Synaptonemal complex formation in hybrids of Lolium temulentum $\times$ Lolium perenne. II. Triploid. Chromosoma, 92, 387-390.
JENKINS, G. (1986). Synaptonemal complex formation in hybrids of Lolium temulentum $\times$ Lolium perenne. III. Tetraploid. Chromosoma, 93, 413-419.

JENKINS, G., WHITE, J. AND PARKER, J. S. (1988). Elimination of multivalents during meiotic prophase in Scilla autumnalis. II. Tetraploid. Genome, 30, 940-946.

KIMBER, G. AND RILEY, R. (1963). The relationships of the diploid progenitors of hexaploid wheat. Can. J. Genet. Cytol., 5, 83-88.

LEGGETT, J. M. (1977). The meiotic behaviour of aneupolyhaploids of the cultivated oat Avena sativa $(2 n=6 x=42)$. Can. J. Genet. Cytol., 19,651-656.

LOIDL, J. (1988). SC formation in some Allium species and a discussion of the significance of $\mathrm{SC}$-associated structures and of the mechanisms for pre-synaptic alignment. Pl. Syst. Evol., 158, 117-131.

MATHER, K. (1943). Statistical analysis in Biology. Methuen (London).

NISHIYAMA, I. AND YABUNO, T. (1975). Meiotic chromosome pairing in two interspecific hybrids and a criticism of the evolutionary relationship of diploid Avena. Jap. J. Genet., $50,443-451$.

RAJHATHY, T. (1963). A standard karyotype of Avena sativa. Can. J. Genet. Cytol, , 5, 127-132.

Rajhathy, T. (1971). The allopolyploid model in Avena. Stadler Symposia, 3, 71-87.

RAJHATHY, T. AND DYCK, P. L. (1963). Chromosomal differentiation and speciation in diploid Avena. II. Karyotype of Avena pilosa. Can. J. Genet. Cytol., 5, 175-179.

RAJHATHY, T. AND THOMAS, H. (1967). Chromosomal differentiation and speciation in diploid Avena. III. Mediterranean wild populations. Can. J. Genet. Cytol., 3, 372-377.

RAJHATHY, T. AND THOMAS, H. (1972). Genetic control of chromosome pairing in hexaploid oats. Nature New Bio$\log y, 239,217-219$.

RAJHATHY, T. AND THOMAS, H. (1974). Cytogenetics of oats (Avena L.). Misc. Pub. Genet. Soc. Canada, 2, 1-91.

RILEY, R. AND CHAPMAN, v. (1958). Genetic control of the cytologically diploid behaviour of hexaploid wheat. Nature, 182, 713-715.

SEARS, E. R. (1941). Chromosome pairing and fertility in hybrids and amphidiploids in the Triticinae. Res. Bull. Missouri Agric. Expt. Sta., 337.

SEARS, E. R. AND OKAMOTO, M. (1958). Intergenomic chromosome relationships in hexaploid wheat (Abstr.). Proc. $X$ Int. Cong. Genet., 2, 258-259.

ThOMAS, H. Cytogenetics of Avena. Oat Monograph, 2nd ed, (In press).

WELLS, B. (1974). A convenient technique for the collection of ultrathin sections. Micron., 5, 79-81. 